\title{
The Intralaminar Thalamic Nuclei Contribute to Remote Spatial Memory
}

\author{
Joëlle Lopez, ${ }^{1}$ Mathieu Wolff, ${ }^{2}$ Lucas Lecourtier, ${ }^{1}$ Brigitte Cosquer, ${ }^{1}$ Bruno Bontempi, ${ }^{2}$ John Dalrymple-Alford, ${ }^{3}$ and \\ Jean-Christophe Cassel ${ }^{1}$ \\ ${ }^{1}$ Laboratoire d'Imagerie et de Neurosciences Cognitives, Université de Strasbourg, Institut Fédératif de Recherche 37 des Neurosciences, Groupement de \\ Recherche, Centre National de la Recherche Scientifique 2905 Neuromem, F-67000 Strasbourg, France, ${ }^{2}$ Centre de Neurosciences Intégratives et Cognitives, \\ Centre National de la Recherche Scientifique Unité Mixte de Recherche 5228, Université de Bordeaux 1, F-33405 Talence Cedex, France, and ${ }^{3}$ Van der Veer \\ Institute for Parkinson's and Brain Research and Department of Psychology, University of Canterbury, Christchurch 8140, New Zealand
}

Recent studies have shown that the anterior (ATN) and lateral thalamic nuclei (including the intralaminar nuclei; ILN/LT) play different roles in memory processes. These nuclei have prominent direct and indirect connections with the hippocampal system and/or the prefrontal cortex and may thus participate in the time-dependent reorganization of memory traces during systems-level consolidation. We investigated whether ATN or ILN/LT lesions in rats influenced acquisition and subsequent retrieval of spatial memory in a Morris water maze. Retrieval was assessed with a probe trial after a short ( $5 \mathrm{~d}$, recent memory) or a long ( $25 \mathrm{~d}$, remote memory) postacquisition delay. The ATN group showed impaired acquisition compared with the Sham controls and ILN/LT groups, which did not differ during acquisition, and exhibited no preference for the target quadrant during the recent or remote memory probe trials. In contrast, probe trial performance in rats with ILN/LT lesions differed according to the age of the memory, with accurate spatial retrieval for the recent memory probe trial but impaired retrieval during the remote memory one. These findings confirm that ATN but not ILN/LT lesions disrupt the acquisition of spatial memory and provide new evidence that the ILN/LT region contributes to remote memory processing. Thus, the lateral thalamus may modulate some aspects of remote memory formation and/or retrieval during the course of systems-level consolidation.

\section{Introduction}

The anterior (ATN) and intralaminar (ILN) thalamic nuclei have been increasingly implicated as key regions in human diencephalic amnesia (Aggleton and Brown, 1999; Gold and Squire, 2006). In rats, ATN lesions produce spatial and nonspatial deficits that are similar to those observed after hippocampal damage (Warburton and Aggleton, 1999; Van Groen et al., 2002; Wolff et al., 2006), and the functional link between these two structures is supported by disconnection studies (Warburton et al., 2001; Henry et al., 2004). In contrast, lesions that include the rostral ILN (i.e., the paracentral, central lateral, and anterior central medial nuclei) and the adjacent lateral mediodorsal thalamic nuclei (ILN/LT lesions) impair performance in tasks that reflect prefrontal cortex function, but have little influence on the acquisition of allocentric spatial memory unless these lesions encroach onto the adjacent ATN (Savage et al., 1997; Bailey and Mair, 2005;

Received Nov. 21, 2008; revised Jan. 22, 2009; accepted Feb. 10, 2009.

This work was supported by the French Agence Nationale de la Recherche (ANR-06-NEURO-027-04), the University Louis Pasteur (J.D.-A.), the Centre National de la Recherche Scientifique, a 3 year fellowship from the French Ministère de l'Enseignement Supérieur et de la Recherche (J.L.), and the Fondation pour la Recherche Médicale (M.W.). We are grateful to 0 . Bildstein, D. Egesi, and G. Edomwonyi for their assistance in animal care, and to Dr. Rodrigue Galani for his help regarding the electronic figure formats.

Correspondence should be addressed to Dr. Jean-Christophe Cassel, Laboratoire d'Imagerie et de Neurosciences Cognitives, Université de Strasbourg, 12 rue Goethe, F-67000 Strasbourg, France. E-mail: jean-christophe.cassel@linc.u-strasbg.fr.

DOI:10.1523/JNEUROSCI.5576-08.2009

Copyright $\odot 2009$ Society for Neuroscience $\quad 0270-6474 / 09 / 293302-05 \$ 15.00 / 0$
Mitchell and Dalrymple-Alford, 2005; Mitchell and DalrympleAlford, 2006; Wolff et al., 2008).

Although the ATN and the ILN differ in their influence on the acquisition of allocentric spatial memory tasks, it is not known whether either region influences consolidation and retrieval of remote spatial memory, which is important for a fuller understanding of their role in memory processes. Both diencephalic regions have prominent neural connections with the prefrontal cortex, especially the medial prefrontal cortex (Berendse and Groenewegen, 1991; Van der Werf et al., 2002), which has been strongly implicated in the expression of remote memory (Frankland et al., 2004; Maviel et al., 2004; Teixeira et al., 2006). We therefore tested the hypothesis that ILN/LT lesions have little impact on spatial memory acquisition in the Morris water maze, yet impair the expression of remote ( $25 \mathrm{~d})$, but not recent ( $5 \mathrm{~d}$ ), spatial memory. While hippocampal dysfunction impairs consolidation in some tasks (Maviel et al., 2004), it fails to spare retention of spatial memory in the water maze (Broadbent et al., 2006), and ATN lesions have similarly failed to spare preoperatively trained spatial memory in this task (Warburton et al., 1999). To confirm the unique influence of ILN/LT lesions, we therefore contrasted the effects of ILN/LT and ATN lesions on performance in recent and remote memory tests after training in a Morris water maze.

\section{Materials and Methods}

Animals and surgery. The study adhered to the regulations specified by the European Committee Council Directive of November 24, 1986 (86/ 
609/EEC) and the French Department of Agriculture (authorization $\mathrm{n}^{\circ} 67-215$ for J.-C.C.). Fifty-nine male Long-Evans rats, 3 months old at the time of surgery, were used. They were housed individually in a quiet room, under a $12 \mathrm{~h} \mathrm{light/dark} \mathrm{cycle} \mathrm{(light} \mathrm{on} \mathrm{at} \mathrm{7:00} \mathrm{A.M.)} \mathrm{with} \mathrm{ad} \mathrm{libitum}$ access to food and water. For surgery, they were anesthetized with sodium pentobarbital (50 mg/kg i.p., $20 \mathrm{~min}$ after atropine, $0.22 \mathrm{mg} / \mathrm{kg}$, i.p.) and secured in a stereotaxic frame (incisor bar $-7.5 \mathrm{~mm}$ ). ATN and ILN/LT lesions were made via slow infusions of sub-microliter volumes of $\mathrm{N}$-methyl-D-aspartate (supplemental Fig. S1, available at www. jneurosci.org as supplemental material). ATN lesions targeted the anterodorsal, anteromedial, and anteroventral thalamic nuclei, while ILN/LT lesions included the centrolateral, paracentral, rostral central medial, and the lateral/paralamellar mediodorsal nuclei. The latter were included because this subregion of the mediodorsal nucleus has similar prefrontal cortex connections to those of the ILN (Mitchell and Dalrymple-Alford, 2005). The sham-operated controls (SHAM) had the infusion needle lowered to either the ATN or ILN/LT coordinates.

After a $10 \mathrm{~d}$ recovery period, the rats first received counterbalanced spatial training on Packard and McGaugh's (1992) two water-maze tasks, which both used visual cues that signaled the varying placement of a hidden platform in one task, but could not be used to identify the fixed platform in the second task. Neither lesion group was impaired on the visual discrimination task, ruling out any nonmnemonic effects of the thalamic lesions on water maze performance. The ILN/LT group was also unimpaired on the spatial task whereas the ATN group showed a marked deficit (data not shown). The current study on recent and remote memory was conducted 2 months later, but in a different water maze placed in a new room with different spatial cues.

Spatial testing in the Morris water maze. The specifications of the water maze and the testing procedures have been described previously (Pereira de Vasconcelos et al., 2006). The first part of training was a four trial session using a visible platform located in the South-East quadrant of the pool, starting randomly from each of the four cardinal points at the edge of the pool. The rats were then given four successive acquisition trials per day (intertrial interval, $10-15 \mathrm{~s}$ ), starting randomly from each of the four cardinal points from the edge of the pool, for eight consecutive days to learn the location of a platform hidden $1 \mathrm{~cm}$ below the water surface in the North-West quadrant. The distance traveled before reaching the platform was computed by a videotracking system (Ethovision, Noldus).

At the end of acquisition, rats from each group were allocated randomly to one of two treatment subgroups and then tested for retention in a probe trial administered either $5 \mathrm{~d}$ (recent memory, $n_{\mathrm{ATN}}=11, n_{\mathrm{ILN} / \mathrm{LT}}=11, n_{\mathrm{SHAM}}=$ 9) or $25 \mathrm{~d}$ postacquisition (remote memory, $n_{\mathrm{ATN}}=10, n_{\mathrm{ILN} / \mathrm{LT}}=10, n_{\mathrm{SHAM}}$ $=8$ ). The platform was removed and all rats were released from the NorthEast start point and given $60 \mathrm{~s}$ to swim. The primary retention measure was the accuracy ratio (Koenig et al., 2008) calculated as follows: time spent in the target quadrant $\left(\mathrm{T}_{\mathrm{QT}}\right)$ multiplied by 3 and divided by the time spent in the other three quadrants $\left(\mathrm{T}_{\mathrm{Q} 1}, \mathrm{~T}_{\mathrm{Q} 2}, \mathrm{~T}_{\mathrm{Q} 3}\right)$. The number of crossings in the target area, corresponding to the size of the platform enlarged by a $10 \mathrm{~cm}$ wide annulus, was analyzed and used as an index of memory precision. Heading vectors were also examined, defined as the deviation (in degrees) of the initial swim segment of the first $25 \mathrm{~cm}$ of a linear swim direction relative to the platform's position.

Histology. Rats received an overdose of sodium pentobarbital (200 $\mathrm{mg} / \mathrm{kg}$, i.p.) and were perfused transcardially with $4 \%$ paraformaldehyde (in $0.1 \mathrm{M} \mathrm{PBS} ; 4^{\circ} \mathrm{C}$ ). Brains were postfixed for $2 \mathrm{~h}$ in the same fixative before cryoprotection for $48 \mathrm{~h}\left(\right.$ at $4^{\circ} \mathrm{C}$ ) in a $20 \%$ sucrose solution (in 0.1 м PBS). The brains were then snap-frozen in isopentane at $-40^{\circ} \mathrm{C}$ and kept at $-80^{\circ} \mathrm{C}$ until coronal sections ( $30 \mu \mathrm{m}$ thick) were made (Reichert Jung cryostat, Frigocut 2800). Estimation of thalamic damage based on cresyl-stained sections was done as described previously (Mitchell and Dalrymple-Alford, 2005) (supplemental Fig. S2, available at www. jneurosci.org as supplemental material). Additional hippocampal sections were stained for acetylcholinesterase (AChE) histochemistry to check whether the lesion procedure had altered the cholinergic innervation of the hippocampus (supplemental Fig. S3, available at www. jneurosci.org as supplemental material).

Statistical analyses. Performance during acquisition was evaluated using a three-way repeated measure ANOVA (day: 1-8; lesion: ATN, ILN/
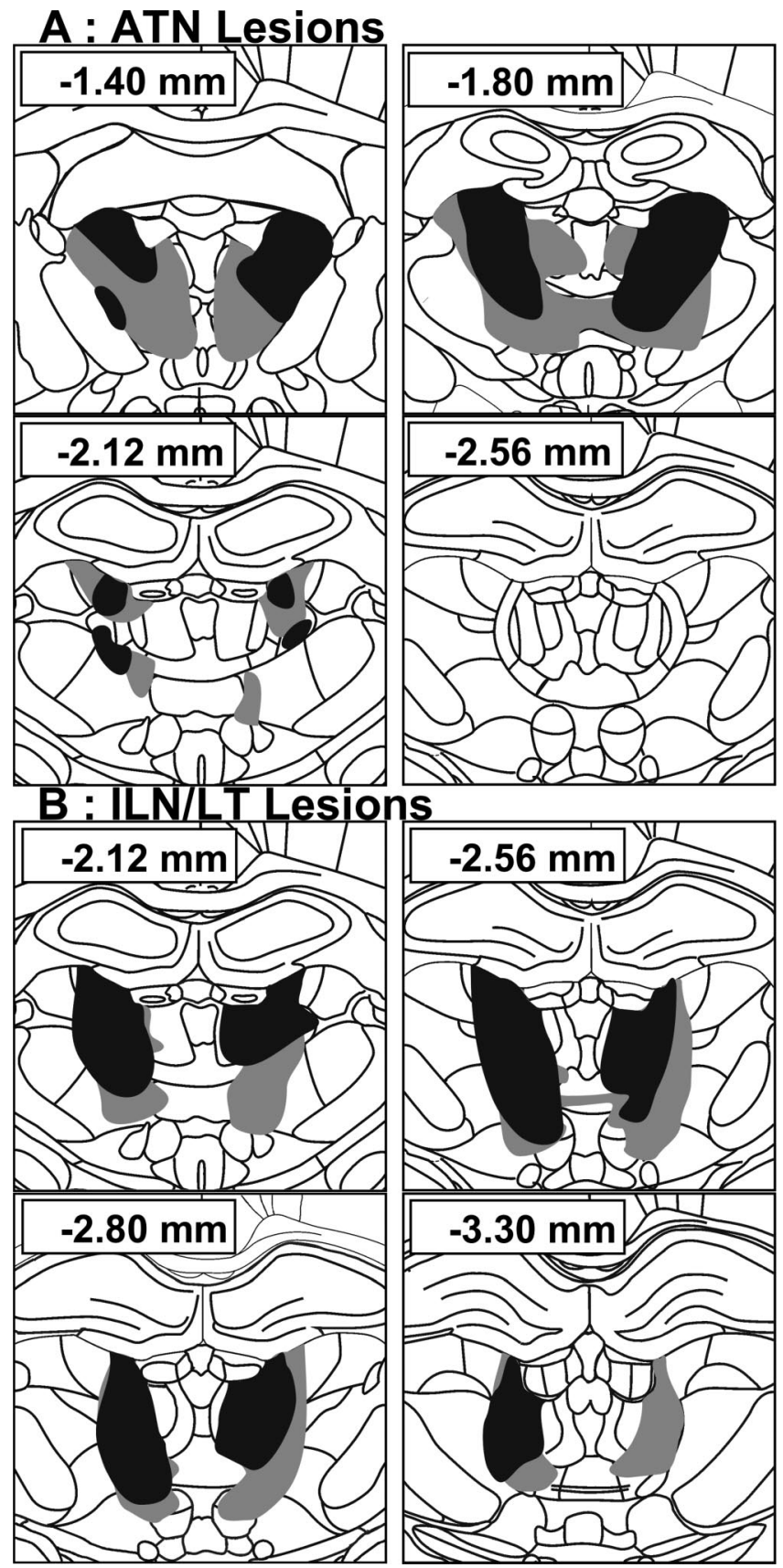

Figure 1. Schematic representation of the smallest (black) and largest (gray) thalamic lesions. $\boldsymbol{A}$, Anterior thalamic nuclei group (ATN). $\boldsymbol{B}$, Intralaminar nucleus/lateral thalamic lesion group (ILN/LT). Numbers (in $\mathrm{mm}$ ) indicate the distance of each section from bregma (according to Paxinos and Watson, 1998).

LT, SHAM; delay: 5 and 25 d). Effects of lesions on retention in each probe trial were analyzed using a one-way ANOVA as well as one-sample $t$ tests to compare the accuracy ratios with chance (i.e., from 1.0). Post hoc comparisons used the Newman-Keuls multiple range test. Values of $p<$ 0.05 were considered significant.

\section{Results}

Placement and extent of the lesions

Figure 1 shows the largest and smallest ATN and ILN/LT lesions in the rats that were included in the behavioral analyses (representative pictures shown in supplemental Fig. S4, available at www.jneurosci.org as supplemental material). Five ATN (two from the $5 \mathrm{~d}$ group, three from the $25 \mathrm{~d}$ group) and three ILN/LT 


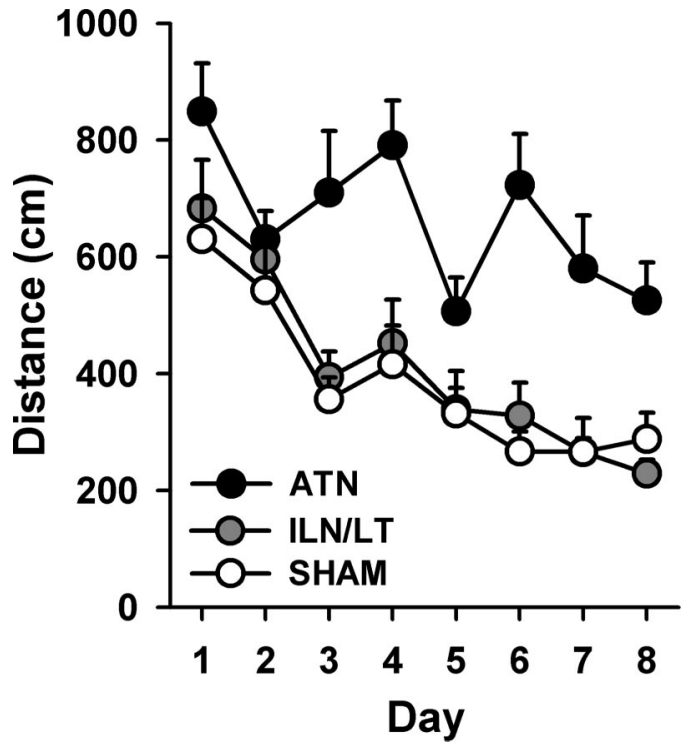

Figure 2. Effects of thalamic lesions on the acquisition of the hidden version of the watermaze task. Mean ( \pm SEM) distances to reach the platform are shown for the ATN, ILN/LT and SHAM groups. ATN rats were impaired.

rats (two from the $5 \mathrm{~d}$ group, one from the $25 \mathrm{~d}$ group) had insufficient damage to the targeted area $(<50 \%)$ and were discarded from the analyses. Final sample sizes were as follows: $5 \mathrm{~d}$ group, $n_{\mathrm{ATN}}=9, n_{\mathrm{ILN} / \mathrm{LT}}=9, n_{\mathrm{SHAM}}=9 ; 25 \mathrm{~d}$ group, $n_{\mathrm{ATN}}=7$, $n_{\mathrm{ILN} / \mathrm{LT}}=9, n_{\mathrm{SHAM}}=8$.

No significant differences in the extent of the ATN and ILN/LT lesions were found between the 5 and $25 \mathrm{~d}$ delay groups $[F<1$, nonsignificant (n.s.) for either lesion]. The damage was comparable to previous work (Mitchell and Dalrymple-Alford, 2006; Wolff et al., 2008) and highly specific to the ATN or ILN/ LT, with minimal damage to the alternate target or other adjacent thalamic nuclei. In the retained 16 ATN rats, there was a median of $75.8 \%$ damage (range, $50.0-94.5 \%$ ) to the ATN, and only 9.9\% damage (range, $1.3-29.4 \%$ ) to the ILN/LT region and $3.8 \%$ damage (range, $0.6-13.7 \%$ ) to the remaining mediodorsal region [lesions of this latter region can induce memory impairments; Mitchell and Dalrymple-Alford (2005)]. In the retained 18 ILN/LT rats, there was a median of $63.7 \%$ damage (range, $50.8-$ $74.3 \%$ ) to the ILN/LT, and only $3.3 \%$ damage (range: $0.0-20.0 \%$ ) to the ATN and $22.2 \%$ damage (range, $5.2-33.5 \%$ ) to the mediodorsal region. Damage to other thalamic structures was minimal (supplemental Fig. S5, available at www.jneurosci.org as supplemental material). ILN/LT lesions had no significant effect on hippocampal AChE staining while ATN lesions marginally affected the ventral hippocampus (supplemental Fig. S6, available at www.jneurosci.org as supplemental material).

\section{Acquisition of the hidden version of the water maze task}

Spatial acquisition of the three groups (ATN, ILN/LT, and SHAM) is shown in Figure 2. While all groups showed improvement over days $\left(F_{(7,322)}=18.5 ; p<0.001\right.$; delay $\times$ lesion $\times$ day interaction: $F_{(14,322)}<1$, n.s.), there was a main effect of lesion $\left(F_{(2,46)}=13.8 ; p<0.001\right)$, with ATN rats swimming significantly longer distances to reach the platform than both the ILN/LT and SHAM groups, which did not differ $(p>0.20)$. Despite reducing their mean path lengths during acquisition, the ATN group's performance remained impaired at the end of acquisition.

\section{Probe trials}

Recent memory (5 d delay)

The mean accuracy ratios during the recent probe trial are shown in Figure $3 A$ (left). There was a significant lesion effect $\left(F_{(2,24)}=\right.$ $6.5 ; p<0.01)$, with the ATN group spending significantly less time in the target area compared with ILN/LT and SHAM groups $(p<0.05$ and $p<0.01$, respectively), which did not differ significantly. For ATN rats, this accuracy ratio was not significantly different from 1.0 (chance level, $t_{8}<1.0$ ). In the two other groups, however, this ratio was significantly higher than 1.0 (ILN/ LT: $t_{8}=4.9, p<0.01$; SHAM: $t_{8}=5.8, p<0.01$ ), showing good recall of the platform's location. There was also a significant Lesion effect in terms of the number of crossings over the target area $\left(F_{(2,24)}=4.7 ; p<0.05\right)$ as the ATN rats crossed the target area significantly less frequently than the rats of the other two groups ( $p<0.05$ for both comparisons) (Fig. 3B, left). These differences in search strategies were visible in the swim paths (Fig. $3 C$, top part). Analysis of the heading vectors (SHAM: $37.4^{\circ} \pm 11.1$; ATN: $47.1^{\circ} \pm 7.8$; ILN/LT: $\left.55.7^{\circ} \pm 7.7\right)$ showed no significant group effect $\left(F_{(2,24)}=1.16\right.$, n.s. $)$. Finally, there were no significant differences among groups regarding thigmotaxic behavior, assessed by measuring the percentage time spent within a $10 \mathrm{~cm}$ annulus from the pool's border $\left(F_{(2,24)}<1.0\right.$, n.s.) (supplemental Fig. S7, available at www.jneurosci.org as supplemental material).

\section{Remote memory (25 d delay)}

The mean accuracy ratios during the remote probe trial are shown in Figure $3 A$ (right). There was a significant main effect of lesion $\left(F_{(2,21)}\right.$ $=8.6 ; p<0.01$ ), which was due to both the ATN and ILN/LTN groups spending significantly less time in the target area compared with SHAM rats ( $p<0.01$ and $p<0.05$, respectively). The accuracy ratio of the ATN group was again not significantly different from chance $\left(t_{6}<1.0\right)$, unlike that of the SHAM group $\left(t_{7}=3.9, p<0.01\right)$. More importantly, the ILN/LT group also showed a poor level of preference for the correct quadrant, although the accuracy ratio was slightly, but significantly, above chance $\left(t_{8}=3.5, p<0.01\right)$. There was also a significant lesion effect for the number of crossings in the target area $\left(F_{(2,21)}=4.4 ; p<0.05\right)$. ATN rats crossed this area significantly less frequently $(p<0.05)$ than SHAM rats (Fig. $3 B$, right). While the ILN/LT rats did not differ from ATN rats on this measure $(p>0.20)$, their reduced tendency to cross the target area just failed to reach significance when compared with SHAM rats $(p=0.062)$. These differences in search strategies were reflected in the swim paths exhibited by the three groups (Fig. $3 C$, bottom). Analysis of the heading vectors (SHAM: $36.5^{\circ} \pm 8.0$; ATN: $46.6^{\circ} \pm 5.6$; ILN/LT: $\left.58.3^{\circ} \pm 9.4\right)$ showed no significant Group effect $\left(F_{(2,21)}=2.01 ; p=\right.$ $0.16)$. Analysis of the thigmotaxic behavior revealed no significant differences among groups $\left(F_{(2,21)}=2.6 ; p=0.1\right)$ (supplemental Fig. S7, available at www.jneurosci.org as supplemental material).

\section{Discussion}

Consistent with previous findings, this study shows that ILN/LT lesions did not affect acquisition of a spatial memory task or its recent retrieval assessed $5 \mathrm{~d}$ after the end of acquisition (Mitchell and Dalrymple-Alford, 2005; 2006; Wolff et al., 2008) (but see Mair et al., 1998; Gibb et al., 2006). In contrast, ILN/LT lesions dramatically impaired performance during the remote memory probe trial. Since these lesions did not affect AChE activity in the hippocampus, it is highly unlikely that the observed impairment was due to an alteration in cholinergic innervation of the hippocampal region. The selective detrimental effect of ILN/LT lesions on remote memory expression suggests a role for the ILN/LT in the processing of remote spatial memory. In contrast, 

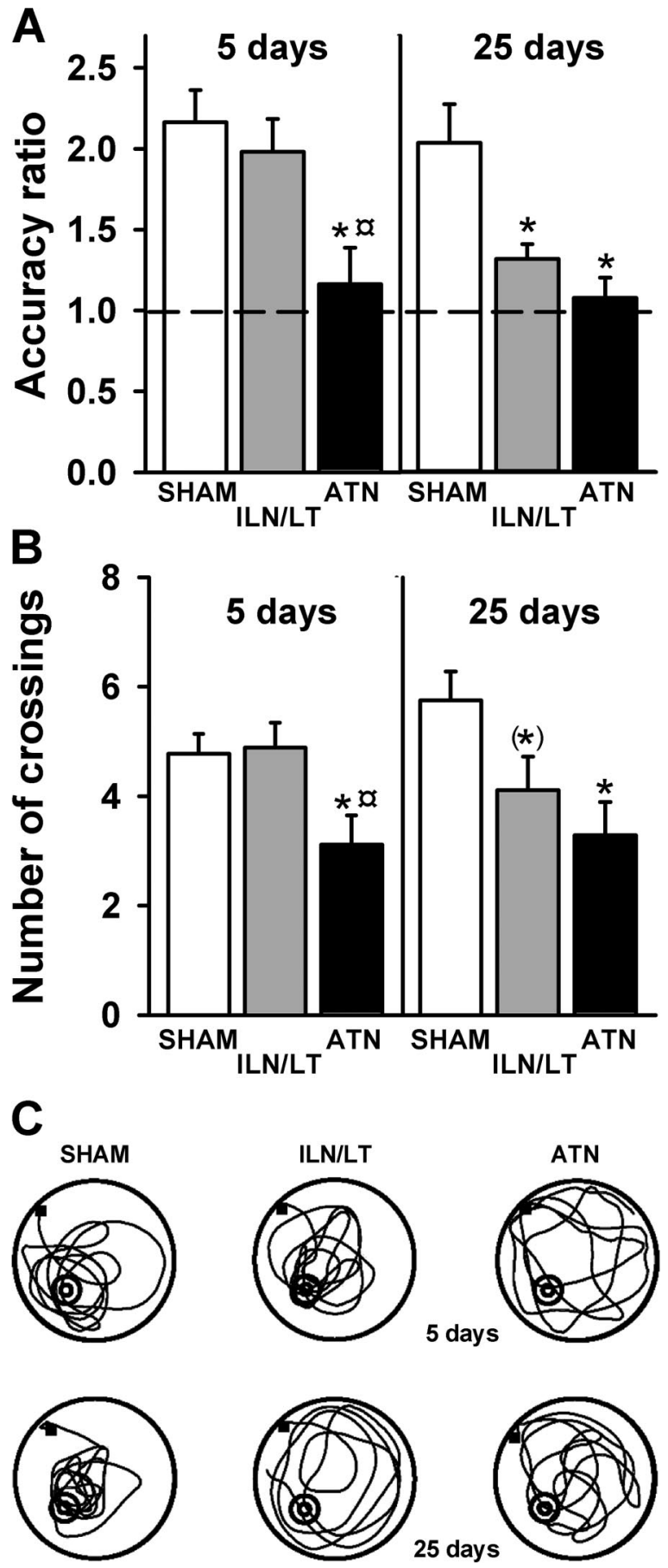

Figure 3. Effects of thalamic lesions on recent and remote spatial memory. $A$, Mean ( \pm SEM) accuracy ratio for the probe trial in the three subgroups tested after a $5 \mathrm{~d}$ (left) or a $25 \mathrm{~d}$ (right) postacquisition delay. Performance is expressed as the accuracy ratio (see Materials and Methods; dashed line: chance). Significantly different from SHAM: ${ }^{*} p<0.01$. Significantly different from ILN/LT: ap $<0.05 . \boldsymbol{B}$, Mean ( \pm SEM) number of crossings in the target area for the probe trial, at the $5 \mathrm{~d}$ (left) and $25 \mathrm{~d}$ (right) postacquisition delays. Significantly different from SHAM: ${ }^{*} p<0.05$. Tendency to differ from SHAM: ${ }^{*} p=0.062$. Significantly different from ILN/LT: ap $<0.05$. C, Representative swim paths in each thalamic group during the $5 \mathrm{~d}$ (recent memory, upper part) and $25 \mathrm{~d}$ (remote memory, lower part) probe trials. The black square indicates the starting point. The position of the platform during acquisition is marked in the lower left quadrant. At both delays, SHAM rats remembered the spatial position of the platform and concentrated their searches in the target quadrant where the platform was located. In contrast, ATN lesions affected spatial exploration at both delays while ILN/LT rats were impaired only during the remote probe trial.
ATN rats were severely impaired at all stages of spatial memory testing (acquisition and retrieval), thus confirming the crucial role played by the ATN in the acquisition of spatial information (Byatt and Dalrymple-Alford, 1996; Aggleton et al., 1996; Bailey and Mair, 2005; Mitchell and Dalrymple-Alford, 2005, 2006; Wolff et al., 2008).

This study provides the first evidence of a selective role of the ILN/LT in the processing of remote spatial memory. The observed remote memory impairment could be explained by the disconnection of the ILN/LT from the anterior cingulate cortex, a subdivision of the medial prefrontal cortex thought to be actively involved in remote memory processing. Indeed, neuronal inactivation of the medial prefrontal cortex impairs retrieval of remote but not recent spatial (Maviel et al., 2004) or contextual fear memories (Frankland et al., 2004). Whether the prefrontal cortex contributes to memory storage per se or exerts a more executive role in integrating and coordinating memory retrieval from other distributed cortical sites remains uncertain (Simons and Spiers, 2003; Frankland and Bontempi, 2005). Consequently, by disrupting prefrontal activity of hippocampal-cortical interactions during the course of the memory consolidation process, ILN/LT lesions may have prevented the formation of memories at the cortical level and/or affected retrieval processes. The ILN/LT also projects to the striatum (Lacey et al., 2007), but it is unlikely that an alteration of this striatal pathway might explain our observed remote memory deficit given the marginal role played by the striatum in either the acquisition or consolidation of place learning (Packard et al., 2001). A procedural memory impairment is equally unlikely as this form of memory is typically very robust and its alteration is likely to cause thigmotaxic behavior, which was not observed here in the lesioned rats.

According to the standard consolidation theory, a memory trace is initially encoded within hippocampal-neocortical networks but extrahippocampal structures such as the prefrontal cortex are thought to become increasingly important over time (Frankland and Bontempi, 2005). The hippocampal-prefrontal pathways are therefore essential for the time-dependent reorganization of memory traces. One of these pathways connects the hippocampus directly to the medial prefrontal cortex, originating from the CA1 region of the hippocampus and the subiculum, and terminating in the prelimbic cortex (Thierry et al., 2000). There are, however, indirect pathways in which thalamic nuclei act as relay stations. Indeed, Groenewegen et al. (1999) described two circuits which are organized into corticostriatal loops, that are themselves connected to the hippocampus. The first circuit starts in the CA1 and the subiculum, and projects to the shell of the nucleus accumbens and to the medial part of the ventral pallidum. These structures in turn project partly to the central medial and paracentral nuclei (part of the ILN/LT aggregate), which then project to the medial prefrontal cortex (Montaron et al., 1996; Groenewegen et al., 1999; Thierry et al., 2000). The second circuit, also starting in the hippocampus, projects toward the core of the nucleus accumbens and then to the dorsomedial part of the substantia nigra pars reticula, before innervating these same thalamic nuclei as well as the medial prefrontal cortex (Montaron et al., 1996; Groenewegen et al., 1999; Thierry et al., 2000). These pathways could thus modulate the direct hippocampalprefrontal pathway that is important for spatial memory processing (Thierry et al., 2000; Floresco and Grace, 2003; Wang and Cai, 2008) and might represent a necessary feedback for remote spatial memory processing.

Due to similarities between the effects of hippocampal and ATN lesions, we also sought to examine the involvement of the ATN in the 
retrieval of recent and remote memory. Just like hippocampal lesions, we found that ATN lesions disrupted the acquisition of the hidden version of the water maze task. Such a deficit confirms previous findings obtained in both a radial maze (Aggleton et al., 1996) and a Morris water maze (Warburton and Aggleton, 1999; Van Groen et al., 2002; Wolff et al., 2008). Given their poor acquisition, it was not surprising that ATN rats performed at chance level during the recent and remote probe trials. While cholinergic innervation of the dorsal hippocampus was not affected, it is noteworthy that AChE staining was mildly reduced in the ventral hippocampus of ATN rats (supplemental Fig. S6, available at www.jneurosci.org as supplemental material), although it is not clear whether this change represents a functional or unintended structural consequence of the ATN lesion. It is unlikely that weaker cholinergic function accounted for the observed acquisition deficit found in ATN rats, as an almost complete cholinergic denervation of the hippocampus has little effect on spatial tasks (Lehmann et al., 2002; Parent and Baxter, 2004).

In conclusion, this study shows that the ILN/LT region plays a selective role in spatial memory processing. While ILN/LT lesions did not interfere with either the acquisition of spatial memory or with its recent expression ( $5 \mathrm{~d}$ delay), we provide evidence that they selectively impaired expression of spatial remote memory by interfering with its formation and/or retrieval ( $25 \mathrm{~d}$ delay). This suggests that the ILN/LT region represents a critical component of an extrahippocampal network involved in the time-dependent reorganization of spatial memory during the course of systemslevel memory consolidation.

\section{References}

Aggleton JP, Brown MW (1999) Episodic memory, amnesia, and the hippocampal-anterior thalamic axis. Behav Brain Sci 22:425-444; discussion 444-489.

Aggleton JP, Hunt PR, Nagle S, Neave N (1996) The effects of selective lesions within the anterior thalamic nuclei on spatial memory in the rat. Behav Brain Res 81:189-198.

Bailey KR, Mair RG (2005) Lesions of specific and non-specific thalamic nuclei affect prefrontal cortex-dependent aspects of spatial working memory. Behav Neurosci 119:410-419.

Berendse HW, Groenewegen HJ (1991) Restricted cortical termination fields of the midline and intralaminar thalamic nuclei in the rat. Neuroscience 42:73-102.

Broadbent NJ, Squire LR, Clark RE (2006) Reversible hippocampal lesions disrupt water maze performance during both recent and remote memory tests. Learn Mem 13:181-191.

Byatt G, Dalrymple-Alford JC (1996) Both anteromedial and anteroventral thalamic lesions impair radial-maze learning in rats. Behav Neurosci 110:1335-1348.

Floresco SB, Grace AA (2003) Gating of hippocampal-evoked activity in prefrontal cortical neurons by inputs from the mediodorsal thalamus and ventral tegmental area. J Neurosci 23:3930-3943.

Frankland PW, Bontempi B (2005) The organization of recent and remote memories. Nat Rev Neurosci 6:119-130.

Frankland PW, Bontempi B, Talton LE, Kaczmarek L, Silva AJ (2004) The involvement of the anterior cingulate cortex in remote contextual fear memory. Science 304:881-883.

Gibb SJ, Wolff M, Dalrymple-Alford JC (2006) Odour-place pairedassociate learning and limbic thalamus: comparison of anterior, lateral and medial thalamic lesions. Behav Brain Res 172:155-168.

Gold JJ, Squire LR (2006) The anatomy of amnesia: neurohistological analysis of three new cases. Learn Mem 13:699-710.

Groenewegen HJ, Galis-de Graaf Y, Smeets, WJ (1999) Integration and segregation of limbic cortico-striatal loops at the thalamic level: an experimental tracing study in rats. J Chem Neuroanat 16:167-185.

Henry J, Petrides M, St-Laurent M, Sziklas V (2004) Spatial conditional associative learning: effects of thalamo-hippocampal disconnection in rats. Neuroreport 15:2427-2431.

Koenig J, Cosquer B, Cassel JC (2008) Activation of septal 5-HT1A recep- tors alters spatial memory encoding, interferes with consolidation, but does not affect retrieval in rats subjected to a water-maze task. Hippocampus 18:99-118.

Lacey CJ, Bolam JP, Magill PJ (2007) Novel and distinct operational principles of intralaminar thalamic neurons and their striatal projections. J Neurosci 27:4374-4384.

Lehmann O, Bertrand F, Jeltsch H, Morer M, Lazarus C, Will B, Cassel JC (2002) 5,7-DHT-induced hippocampal 5-HT depletion attenuates behavioural deficits produced by 192 IgG-saporin lesions of septal cholinergic neurons in the rat. Eur J Neurosci 15:1991-2006.

Mair RG, Burk JA, Porter MC (1998) Lesions of the frontal cortex, hippocampus, and intralaminar thalamic nuclei have distinct effects on remembering in rats. Behav Neurosci 112:772-792.

Maviel T, Durkin TP, Menzaghi F, Bontempi B (2004) Sites of neocortical reorganization critical for remote spatial memory. Science 305:96-99.

Mitchell AS, Dalrymple-Alford JC (2005) Dissociable memory effects after medial thalamus lesions in the rat. Eur J Neurosci 22:973-985.

Mitchell AS, Dalrymple-Alford JC (2006) Lateral and anterior thalamic lesions impair independent memory systems. Learn Mem 13:388-396.

Montaron MF, Deniau JM, Menetrey A, Glowinski J, Thierry AM (1996) Prefrontal cortex inputs of the nucleus accumbens-nigro-thalamic circuit. Neuroscience 71:371-382.

Packard MG, McGaugh JL (1992) Double dissociation of fornix and caudate nucleus lesions on acquisition of two water maze tasks: further evidence for multiple memory systems. Behav Neurosci 106:439-446.

Packard MG, Vecchioli SF, Schroeder JP, Gasbarri A (2001) Taskdependent role for dorsal striatum glutamate receptors in memory. Learn Mem 8:96-103.

Parent MB, Baxter MG (2004) Septohippocampal acetylcholine: involved in but not necessary for learning and memory? Learn Mem 11:9-20.

Paxinos G, Watson C (1998) The rat brain in stereotaxic coordinates, Ed 4. San Diego: Academic.

Pereira de Vasconcelos A, Klur S, Muller C, Cosquer B, Lopez J, Certa U, Cassel JC (2006) Reversible inactivation of the dorsal hippocampus by tetrodotoxin or lidocaine: a comparative study on cerebral functional activity and motor coordination in the rat. Neuroscience 141:1649-1663.

Savage LM, Sweet AJ, Castillo R, Langlais PJ (1997) The effects of lesions to thalamic lateral internal medullary lamina and posterior nuclei on learning, memory and habituation in the rat. Behav Brain Res 82:133-147.

Simons JS, Spiers HJ (2003) Prefrontal and medial temporal lobe interactions in long-term memory. Nat Rev Neurosci 4:637-648.

Teixeira CM, Pomedli SR, Maei HR, Kee N, Frankland PW (2006) Involvement of the anterior cingulated cortex in the expression of spatial memory. J Neurosci 26:7555-7564.

Thierry AM, Gioanni Y, Dégénétais E, Glowinski J (2000) Hippocampoprefrontal cortex pathway: anatomical and electrophysiological characteristics. Hippocampus 10:411-419.

Van der Werf YD, Witter MP, Groenewegen HJ (2002) The intralaminar and midline nuclei of the thalamus. Anatomical and functional evidence for participation in processes of arousal and awareness. Brain Res Rev 39:107-140.

van Groen T, Kadish I, Michael Wyss J (2002) Role of the anterodorsal and anteroventral nuclei of the thalamus in spatial memory in the rat. Behav Brain Res 132:19-28.

Wang GW, Cai JX (2008) Reversible disconnection of the hippocampalprelimbic cortical circuit impairs spatial learning but not passive avoidance learning in rats. Neurobiol Learn Mem 90:365-373.

Warburton EC, Aggleton JP (1999) Differential deficits in the Morris water maze following lesions of the anterior thalamus and fornix transection. Behav Brain Res 98:27-38.

Warburton EC, Baird A, Morgan A, Muir JL, Aggleton JP (2001) The conjoint importance of the hippocampus and anterior thalamic nuclei for allocentric spatial learning: evidence from a disconnection study in the rat. J Neurosci 21:7323-7330.

Wolff M, Gibb SJ, Dalrymple-Alford JC (2006) Beyond spatial memory: the anterior thalamus and memory for the temporal order of a sequence of odor cues. J Neurosci 26:2907-2913.

Wolff M, Gibb SJ, Cassel JC, Dalrymple-Alford JC (2008) Anterior but not intralaminar thalamic nuclei support allocentric spatial memory. Neurobiol Learn Mem 90:71-80. 